\section{Estructura Factorial y valores de referencia del WHOQoL-Bref en población adulta chilena}

\author{
ALFONSO URZÚA M. ${ }^{1, a}$, ALEJANDRA CAQUEO-URÍZAR²,b
}

\section{Factorial structure of WHOQoL-Bref quality of life questionnaire}

Background: The real value of quality of life instruments must be tested in local populations before recommending their widespread use. Aim: To assess the factorial structure of the quality of life questionnaire proposed by the World Health Organization (WHO), WHOQoL-Bref. Material and Methods: The questionnaire was answered by 2016 individuals aged between 20 and 59 years, from diverse public and social organizations of a city in Northern Chile. The confidence and factorial structure of the instrument were evaluated. Results: The internal consistency of the instrument, according to Cronbach's alpha was 0.89. The adjustment of values to a model with four factors proposed by the WHO was moderate. The root mean square (RMS) and root mean square error of approximation (RMSEA) values of 0.043 and 0.061, indicate a good adjustment of the model. Conclusions: Although an absolute adjustment for the theoretical four factor model is not supported by these results, the WHOQoL-Bref is able to discriminate the perception of quality of life and the influence of age, gender and disease on this perception.

(Rev Med Chile 2013; 141: 1547-1554)

Key words: Quality of life; Questionnaires; World Health Organization.

\author{
'Línea de Investigación Salud, \\ Bienestar y Calidad de Vida. Escuela \\ de Psicología, Universidad Católica \\ del Norte, Avenida Angamos 0610, \\ Antofagasta, Chile. \\ 2Departamento de Psicología, \\ Universidad de Tarapacá. \\ aPsicólogo, Doctor en Psicología Clínica \\ y de la Salud, Magíster en Salud \\ Pública. \\ 'Psicóloga, Doctora en Psicología \\ Clínica y de la Salud, Magíster en \\ Psicopatología Infanto-juvenil. \\ Este artículo es producto de un \\ estudio financiado por la Comisión \\ Nacional de Investigación Científica \\ y Tecnológica del Gobierno de \\ Chile-CONICYT, a través del proyecto \\ FONDECYT 11100090 y por el \\ Convenio de Desempeño UTA- \\ MINEDUC, quienes no tuvieron \\ influencia en el diseño del estudio, \\ recolección, análisis o interpretación \\ de los datos, ni en la preparación, \\ revisión o aprobación del manuscrito. \\ Recibido el 19 de agosto de 2013, \\ aceptado el 28 de noviembre de 2013. \\ Correspondencia: \\ Dr. Alfonso Urzúa M. \\ Escuela de Psicología, Universidad \\ Católica del Norte, Avenida Angamos \\ 0610, Antofagasta, Chile \\ Celular: (9)72173890. \\ E-mail: alurzua@ucn.cl
}

L a necesidad de incorporar nuevos indicadores en salud pública con una mirada más comprensiva al proceso de salud - enfermedad, ha permitido la incorporación en el campo de la evaluación sanitaria de nuevos conceptos, como es el caso de la Calidad de Vida (CV). La Organización Mundial de la Salud (OMS) la define como la percepción individual de la posición en la vida en el contexto de la cultura y sistema de valores en el cual se vive y su relación con las metas, expectativas, estándares e intereses ${ }^{1}$. Operacionalmente, puede entenderse como el nivel percibido de bienestar derivado de la evaluación que realiza cada persona de elementos objetivos y subjetivos en distintas dimensiones de su vida ${ }^{2}$. La OMS ha remarcado la importancia de considerar la evaluación de la CV por los propios pacientes en las decisiones de tratamiento, la aprobación de nuevas investigaciones farmacéuticas y en la evaluación de programas y focalización de recursos ${ }^{3}$.

A pesar del creciente reconocimiento de la CV como un indicador de evaluación, aún no existen acuerdos unánimes acerca de lo que se define por $\mathrm{CV}$, las dimensiones que la comprenden y los factores que pueden incidir en su evaluación. Este hecho ha repercutido directamente en la diversidad de evaluaciones y la dificultad en el uso de pruebas comunes que permitan establecer comparaciones entre poblaciones similares.

Surge así la necesidad de investigar con definiciones explícitas e instrumentos que permitan comparar distintas poblaciones con similares características a fin de profundizar, por ejemplo, en la posible relación, asociación o rol modulador 
que pudiesen tener otras variables sobre la calidad de vida en diferentes países y contextos. Tal como lo señala la OMS estas mediciones transculturales permiten realizar comparaciones epidemiológicas a nivel internacional, facilitando la comprensión de la enfermedad y el desarrollo de métodos de tratamiento, contribuyendo al avance de una equivalencia conceptual, semántica, funcional y psicométrica de las mediciones en calidad de vida en diversas culturas ${ }^{3}$.

En este contexto, a partir de la década 1990-99, la Organización Mundial de la Salud diseñó de manera colaborativa un instrumento de evaluación en calidad de vida, el WHOQoL-100, siendo las preguntas generadas con base en la información recogida desde los propios pacientes, sus familias y los profesionales de la salud a través de grupos focales, existiendo diversas publicaciones sobre sus características psicométricas ${ }^{4-6}$.

Los análisis de la prueba piloto permitieron, utilizando diversos criterios, reducir el número de ítems a 26, creando el WHOQoL-Bref ${ }^{7-8}$.

Las características psicométricas de este instrumento han sido reportadas en diversas investigaciones, tanto en sus primeras validaciones ${ }^{7-9}$, como en su posterior aplicación a grupos específicos ${ }^{10-18}$ o determinados idiomas y países ${ }^{19-21}$.

Sobre la validación de este instrumento en el idioma castellano, esta ha sido reportada por Lucas en la ciudad de Barcelona en España ${ }^{22}$. Pese a que en Chile ha sido utilizado en diversas investigaciones $^{23-28}$, sólo se ha reportado sus propiedades psicométricas en población adulto mayor, donde se mantiene la estructura factorial original ${ }^{29}$. En este contexto, el presente estudio tuvo por objetivo evaluar la estructura factorial del WHOQoL-Bref en distintos rangos de edad en población adulta. En forma adicional, se reportan valores de referencia para cada uno de los subgrupos, considerando la necesidad de contar con información práctica que permita la evaluación de adultos con base al valor de su grupo poblacional de referencia, establecer líneas base para programas de intervención, y realizar estudios longitudinales en $\mathrm{CV}$.

\section{Material y Método}

Esta investigación se enmarca en la metodología cuantitativa. El diseño es de tipo no experimental, analítico transversal.

\section{Participantes}

La muestra estuvo formada por 2.186 personas, evaluadas en el marco del proyecto FONDECYT 1110090 "Procesos socio-cognitivos vinculados a la evaluación de la calidad de vida", ejecutado durante los años 2011 y 2012 en la ciudad de Antofagasta, Chile.

El tipo de muestreo fue no probabilístico por conveniencia, de tipo estratificado, intentando cuidar similar distribución por sexo. Se consideraron como criterios de exclusión para participar en la investigación la presencia de deterioro físico o mental evidente y estados avanzados de deterioro cognitivo que impidieran responder los cuestionarios. Los encuestadores fueron parte de un grupo estable de investigación y contaban con entrenamiento en procedimientos de recolección de información.

Los participantes fueron reclutados en organizaciones públicas y sociales de la ciudad, tales como universidades, empresas privadas, instituciones gubernamentales, salas de espera de INP, FONASA, bancos, etc. Del total de encuestados, 310 personas $(15,5 \%)$ reportaron vivir con alguna enfermedad crónica. Las patologías prevalentes fueron hipertensión arterial (23\%), diabetes mellitus (17\%), hipotiroidismo (10\%), músculoesqueléticas $(9,0 \%)$ y asma $(8 \%)$. No existió corroboración clínica de la enfermedad crónica, se consideró sólo el autoreporte de enfermedad.

\section{Instrumento}

El WHOQoL- Bref está compuesto por 26 preguntas, de las cuales, una inquiere sobre la calidad de vida general, una sobre satisfacción con la salud y las 24 restantes se agrupan en cuatro dominios de calidad de vida, siendo estos salud física (7 preguntas), salud psicológica (6 preguntas), relaciones sociales ( 3 preguntas) y ambiente ( 8 preguntas).

Cada encuestado debe responder a cada faceta en categorías cuyo puntaje oscila entre 1 y 5 puntos (Escala Likert). Los puntajes de los dominios luego son convertidos según una tabla de corrección de puntajes debido a la desigualdad en el número de ítems, teniendo la posibilidad de convertirse en un rango de 0 a 100 o de 4 a $20^{30}$. En esta investigación hemos utilizado la puntuación de 0 a 100, para efectos de su comparación con los valores normativos propuestos para la escala $^{31}$. Se considera que a mayor puntuación mejor CV. 
Estructura y valores del WHOQoL-Bref en adultos chilenos - A. Urzúa et al

\section{Procedimientos}

La investigación fue evaluada y aprobada por el Comité de Ética de la Universidad Católica del Norte y por el Comité de Bioética del Fondo Nacional de Desarrollo Científico y Tecnológico (FONDECYT), siguiendo los procedimientos éticos concordantes con la Declaración de Helsinki.

Los cuestionarios fueron aplicados por entrevistadores capacitados por el investigador principal. Las aplicaciones se realizaron en forma individual, previa firma del consentimiento informado. El tiempo promedio de aplicación fue de aproximadamente $45 \mathrm{~min}$.

\section{Resultados}

\section{Participantes}

De las 2.016 personas evaluadas, $49,9 \%$ eran mujeres. El rango de edad estuvo entre 20 y 59 años. El promedio de edad para la muestra total fue de 36,19 años ( $D E=12,01$ años); para las mujeres de 36,11 años $(\mathrm{DE}=12,03)$ y para los hombres de 36,27 años (DE $=11,99)$. La Tabla 1 muestra la distribución de la muestra por rangos de edad, sexo y presencia de enfermedad crónica (EC).

\section{Calidad de vida}

Tal como se puede apreciar en la Figura 1, el dominio físico fue el mejor evaluado tanto en hombres como mujeres, en tanto el ambiental fue el que obtuvo el menor puntaje en ambos sexos. Las mujeres obtuvieron puntajes significativamente mayores que los hombres en la dimensión física $\left(\mathrm{t}_{(2012)}=2,023 ; \mathrm{p}<, 001\right)$, psicológica $\left(\mathrm{t}_{(2012,857)}=\right.$ $2,250 ; \mathrm{p}<, 05)$ y ambiental $\left(\mathrm{t}_{(2014)}=2,087 ; \mathrm{p}<, 05\right)$.

Las diferencias también fueron estadísticamente significativas al separar la muestra entre aquellos que reportaban o no enfermedad crónica, siendo

Tabla 1. Distribución de los participantes por rango de edad, sexo y reporte de enfermedad crónica

\begin{tabular}{|cccccccccc|}
\hline $\begin{array}{c}\text { Rango edad } \\
\text { años }\end{array}$ & \multicolumn{2}{c}{ Muestra Total } & \multicolumn{2}{c}{ Hombres } & \multicolumn{2}{c|}{ Mujeres } & \multicolumn{3}{c|}{ Presencia EC } \\
Frec & $\%$ & Frec & \% & Frec & $\%$ & Frec & \% \\
\hline $20-29$ & 792 & 39,30 & 394 & 49,7 & 398 & 50,3 & 61 & 7,7 \\
$30-39$ & 401 & 19,88 & 198 & 49,4 & 203 & 50,6 & 50 & 12,5 \\
\hline $40-49$ & 448 & 22,22 & 230 & 51,3 & 218 & 48,7 & 85 & 19,0 \\
\hline $50-59$ & 375 & 18,60 & 188 & 50,1 & 187 & 49,9 & 114 & 30,4 \\
\hline Total & 2.016 & 100,0 & 1.010 & 50,10 & 1.006 & 49,90 & 310 & 15,4 \\
\hline
\end{tabular}

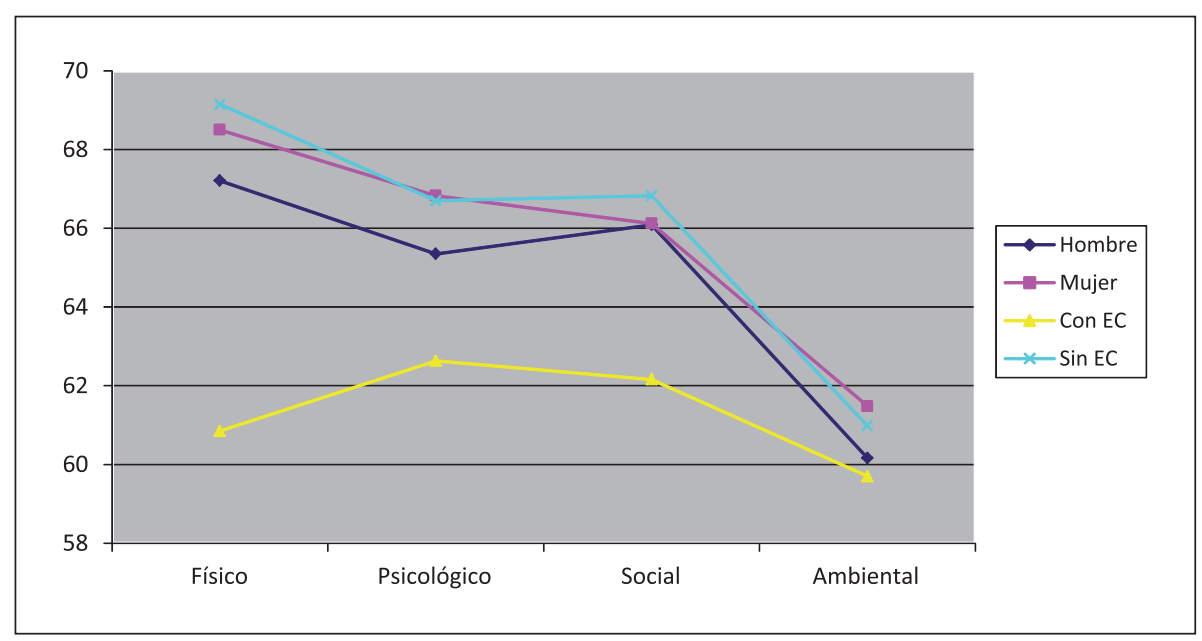

Figura 1. Medias obtenidas por dominios por sexo y reporte/no reporte de enfermedad crónica. 
Tabla 2. Medidas de tendencia central por rango de edad, sexo y presencia/ausencia de enfermedad crónica

\begin{tabular}{|c|c|c|c|c|c|c|c|c|c|c|}
\hline \multirow{2}{*}{$\begin{array}{c}\text { Rango } \\
\text { edad años }\end{array}$} & & \multirow[b]{2}{*}{$\mathbf{n}$} & \multicolumn{2}{|c|}{ Físico } & \multicolumn{2}{|c|}{ Psicológico } & \multicolumn{2}{|c|}{ Social } & \multicolumn{2}{|c|}{ Ambiental } \\
\hline & & & M & DE & $\mathbf{M}$ & DE & $\mathbf{M}$ & DE & $\mathbf{M}$ & DE \\
\hline \multirow[t]{5}{*}{$20-29$} & Hombre & 391 & 67,37 & 13,91 & 64,57 & 14,87 & 68,53 & 16,95 & 59,93 & 14,38 \\
\hline & Mujer & 397 & 69,71 & 13,12 & 67,00 & 14,70 & 68,47 & 17,97 & 61,65 & 13,80 \\
\hline & Con EC & 61 & 61,87 & 15,68 & 62,11 & 16,97 & 68,34 & 20,17 & 61,93 & 15,89 \\
\hline & $\operatorname{Sin} E C$ & 720 & 69,08 & 13,27 & 66,05 & 14,63 & 68,48 & 17,25 & 60,56 & 13,90 \\
\hline & Total & 788 & 68,55 & 13,57 & 65,79 & 14,82 & 68,50 & 17,46 & 60,80 & 14,11 \\
\hline \multirow[t]{5}{*}{$30-39$} & Hombre & 198 & 67,86 & 15,00 & 65,66 & 14,39 & 66,55 & 17,81 & 59,54 & 15,46 \\
\hline & Mujer & 203 & 69,84 & 12,55 & 68,93 & 14,24 & 67,51 & 17,00 & 62,69 & 13,22 \\
\hline & Con EC & 50 & 63,80 & 14,98 & 66,22 & 14,97 & 65,02 & 17,79 & 60,34 & 17,11 \\
\hline & $\operatorname{Sin} E C$ & 348 & 69,64 & 13,56 & 67,56 & 14,33 & 67,35 & 17,30 & 61,31 & 14,08 \\
\hline & Total & 401 & 68,86 & 13,83 & 67,31 & 14,39 & 67,04 & 17,39 & 61,14 & 14,44 \\
\hline \multirow[t]{5}{*}{$40-49$} & Hombre & 230 & 65,71 & 15,96 & 65,02 & 14,56 & 64,80 & 17,38 & 59,81 & 13,89 \\
\hline & Mujer & 218 & 68,56 & 12,73 & 67,25 & 13,55 & 66,08 & 17,21 & 61,40 & 13,43 \\
\hline & Con EC & 85 & 60,29 & 14,47 & 63,25 & 15,26 & 62,04 & 18,91 & 59,08 & 15,18 \\
\hline & $\operatorname{Sin} E C$ & 361 & 68,73 & 14,12 & 66,76 & 13,79 & 66,27 & 16,61 & 60,94 & 13,28 \\
\hline & Total & 448 & 67,10 & 14,53 & 66,10 & 14,10 & 65,42 & 17,29 & 60,58 & 13,67 \\
\hline \multirow[t]{5}{*}{ 50-59 } & Hombre & 187 & 67,99 & 16,54 & 67,08 & 15,47 & 62,07 & 18,83 & 61,75 & 13,97 \\
\hline & Mujer & 186 & 64,38 & 15,69 & 63,70 & 15,82 & 59,65 & 18,05 & 59,89 & 15,27 \\
\hline & Con EC & 113 & 59,43 & 16,41 & 60,85 & 16,32 & 57,68 & 17,11 & 58,66 & 14,96 \\
\hline & $\operatorname{Sin} E C$ & 253 & 69,31 & 15,36 & 67,29 & 15,21 & 62,22 & 19,09 & 61,78 & 14,56 \\
\hline & Total & 373 & 66,20 & 16,20 & 65,39 & 15,72 & 60,86 & 18,46 & 60,82 & 14,65 \\
\hline \multirow[t]{5}{*}{ Total } & Hombre & 1006 & 67,21 & 15,12 & 65,35 & 14,83 & 66,09 & 17,71 & 60,16 & 14,42 \\
\hline & Mujer & 1004 & 68,50 & 13,58 & 66,83 & 14,66 & 66,12 & 17,90 & 61,48 & 13,90 \\
\hline & Con EC & 309 & 60,85 & 15,53 & 62,63 & 15,98 & 62,16 & 18,70 & 59,70 & 15,54 \\
\hline & $\operatorname{Sin} E C$ & 1682 & 69,15 & 13,84 & 66,70 & 14,48 & 66,83 & 17,53 & 60,98 & 13,91 \\
\hline & Total & 2014 & 67,85 & 14,38 & 66,09 & 14,76 & 66,10 & 17,80 & 60,82 & 14,17 \\
\hline
\end{tabular}

las medias de estos últimos significativamente mayores en los dominios de salud física $\left(\mathrm{t}_{(1993)}=\right.$ $-9,513 ; \mathrm{p}<.001)$, salud psicológica $\left(\mathrm{t}_{(1994)}=-4,471\right.$; $\mathrm{p}<.001)$ y social $\left(\mathrm{t}_{(1995)}=-4,269 ; \mathrm{p}<.001\right)$.

La Tabla 2 muestra las medidas de tendencia central, en tanto media (M) y desviación estándar (DE) por cada uno de los rango de edad, separados por sexo y presencia/ausencia de enfermedad crónica.

Tal como se puede observar en la Figura 2, a medida que aumenta la edad, en las mujeres el puntaje tiende a disminuir en todos los dominios evaluados, en tanto en los hombres existe un ligero incremento en el rango de 50 a 59 años en los dominios físico, psicológico y ambiental.
Este decremento es significativo en las mujeres, tanto en los dominios físico $\left(\mathrm{F}_{(3,1001)}=7,578\right.$; $\mathrm{p}=0,000)$, donde la media del rango entre $50 \mathrm{y}$ 59 años fue significativamente menor que los grupos de edad menor $(\mathrm{p}=0,000)$, en el psicológico $\left.\mathrm{F}_{(3,1002)}=4,344 ; \mathrm{p}=0,005\right)$, donde la media del rango de edad entre 30 y 39 años fue significativamente mayor que la del rango de 50 a 59 años $(\mathrm{p}=0,003)$ y social $\left(\mathrm{F}_{(3,1002)}=11,160 ; \mathrm{p}=0,000\right)$, donde la media del rango de 50 a 59 años fue significativamente inferior a los grupos de menor edad $(p=0,000)$. En los hombres sólo fue significativa la diferencia de medias entre los rangos de edad en el dominio social $\left(\mathrm{F}_{(3,1006)}=6,274 ; \mathrm{p}=0,000\right)$, donde la media del grupo entre 20 y 29 años fue 


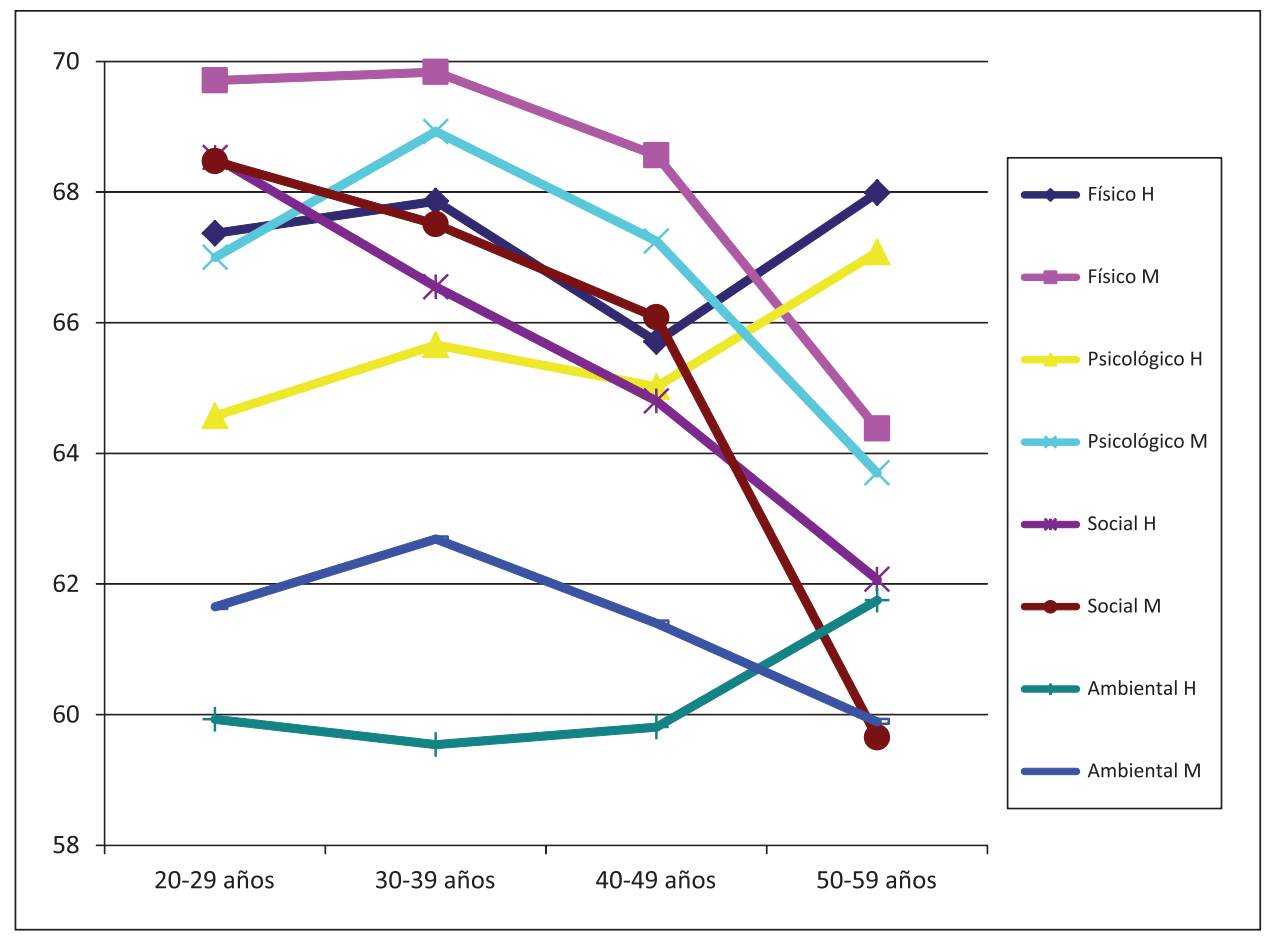

Figura 2. Medias obtenidas por dominios por rango de edad. significativamente mayor que la del rango entre 50 y 59 años $(\mathrm{p}=0,000)$.

La Tabla 3 muestra la distribución en percentiles de los valores obtenidos en cada uno de los dominios del WHOQoL-Bref, según sexo y rango de edad.

\section{Confiabilidad}

El alfa de Cronbach para la escala total fue de .89. Para las dimensiones que constituyen la escala fueron de .71 para la dimensión salud física, .75 para la dimensión psicológica, .63 para la dimensión social y de .76 para la dimensión ambiental.

\section{Estructura factorial}

El ajuste de los valores al modelo de cuatro factores propuestos por la OMS fue moderado. Se espera que los indicadores de ajuste CFI, NFI, GFI y AGFI, sean iguales o mayores a 0,95 ; valor que no fue alcanzado en ninguno de estos ${ }^{32}$. Pese a esto, los valores obtenidos para los indicadores RMRS (el cual se espera sea próximo a cero) y RMSEA (se espera sean menores a 0,08), indican un buen ajuste del modelo. Los valores pueden ser observados en la Tabla 4.

\section{Discusión}

El cuestionario WHOQoL-Bref es uno de los instrumentos más utilizados en investigación sanitaria en el campo de la evaluación de la CV en población general o bien en patologías específicas, pese a esto, en su versión en castellano existe poca información acerca de su validez de constructo, en tanto estructura factorial.

Los resultados obtenidos, aun cuando no indican un ajuste absoluto de los datos al modelo teórico de cuatro factores, indican que el instrumento es capaz de discriminar la CV percibida y el efecto que puede tener tanto el sexo, como la edad y la presencia de una enfermedad crónica, aportando insumos a su validez discriminante. Estos datos son relevantes, en tanto aportan evidencia a la necesidad de disminuir las inequidades existentes en salud analizando los datos desde una perspectiva de género, así como por rango de edad.

Los resultados, asimismo, permiten apreciar como la CV disminuye a medida que las personas se hacen mayores, especialmente en las mujeres, $y$ que la presencia de una enfermedad crónica disminuye la $\mathrm{CV}$ de las personas, independientemente 
Estructura y valores del WHOQoL-Bref en adultos chilenos - A. Urzúa et al

Tabla 3. Valores de referencia de los dominios del WHOQoL-Bref a partir de una muestra de adultos según sexo y rango de edad

\begin{tabular}{|c|c|c|c|c|c|c|c|c|}
\hline \multirow{2}{*}{$\begin{array}{l}\text { Deciles por } \\
\text { dimensión }\end{array}$} & \multicolumn{4}{|c|}{ Hombres } & \multicolumn{4}{|c|}{ Mujeres } \\
\hline & $\begin{array}{l}20-29 \\
(391)\end{array}$ & $\begin{array}{l}30-39 \\
(198)\end{array}$ & $\begin{array}{l}40-49 \\
(230)\end{array}$ & $\begin{array}{l}50-59 \\
(139)\end{array}$ & $\begin{array}{l}20-29 \\
(397)\end{array}$ & $\begin{array}{l}30-39 \\
(203)\end{array}$ & $\begin{array}{l}40-49 \\
(218)\end{array}$ & $\begin{array}{l}50-59 \\
(186)\end{array}$ \\
\hline \multicolumn{9}{|l|}{ Físico } \\
\hline P5 & 44,00 & 44,00 & 38,00 & 44,00 & 44,00 & 50,00 & 44,00 & 38,00 \\
\hline P10 & 50,00 & 50,00 & 44,00 & 44,00 & 56,00 & 56,00 & 56,00 & 44,00 \\
\hline P20 & 56,00 & 56,00 & 56,00 & 56,00 & 63,00 & 56,00 & 56,00 & 52,40 \\
\hline P30 & 63,00 & 63,00 & 56,00 & 56,00 & 63,00 & 63,00 & 63,00 & 56,00 \\
\hline P40 & 63,00 & 63,00 & 63,00 & 63,00 & 69,00 & 69,00 & 63,00 & 63,00 \\
\hline P50 & 69,00 & 69,00 & 63,00 & 69,00 & 69,00 & 69,00 & 69,00 & 63,00 \\
\hline P60 & 69,00 & 69,00 & 69,00 & 69,00 & 69,00 & 71,40 & 69,00 & 69,00 \\
\hline P70 & 75,00 & 75,00 & 75,00 & 81,00 & 75,00 & 79,80 & 75,00 & 69,00 \\
\hline P80 & 81,00 & 81,00 & 81,00 & 81,00 & 81,00 & 81,00 & 81,00 & 75,00 \\
\hline P90 & 88,00 & 88,00 & 88,00 & 94,00 & 88,00 & 88,00 & 88,00 & 88,00 \\
\hline P95 & 88,00 & 94,00 & 94,00 & 94,00 & 94,00 & 92,80 & 94,00 & 91,00 \\
\hline \multicolumn{9}{|l|}{ Psicológico } \\
\hline P5 & 44,00 & 43,70 & 44,00 & 38,00 & 44,00 & 44,00 & 44,00 & 31,00 \\
\hline P10 & 44,00 & 44,00 & 44,00 & 44,00 & 50,00 & 50,00 & 44,00 & 44,00 \\
\hline P20 & 50,00 & 56,00 & 56,00 & 56,00 & 56,00 & 56,00 & 56,00 & 50,00 \\
\hline P30 & 56,00 & 56,00 & 56,00 & 63,00 & 56,00 & 63,00 & 63,00 & 56,00 \\
\hline P40 & 63,00 & 63,00 & 63,00 & 63,00 & 63,00 & 69,00 & 66,60 & 63,00 \\
\hline P50 & 69,00 & 69,00 & 69,00 & 69,00 & 69,00 & 69,00 & 69,00 & 63,00 \\
\hline P60 & 69,00 & 69,00 & 69,00 & 69,00 & 69,00 & 75,00 & 69,00 & 69,00 \\
\hline P70 & 69,00 & 75,00 & 69,00 & 75,00 & 75,00 & 75,00 & 75,00 & 69,00 \\
\hline P80 & 75,00 & 81,00 & 79,80 & 81,00 & 81,00 & 81,00 & 81,00 & 75,00 \\
\hline P90 & 81,00 & 81,00 & 81,00 & 88,00 & 81,00 & 88,00 & 81,00 & 81,00 \\
\hline P95 & 88,00 & 88,30 & 90,70 & 94,00 & 88,00 & 94,00 & 88,00 & 88,00 \\
\hline \multicolumn{9}{|l|}{ Social } \\
\hline P5 & 44,00 & 44,00 & 31,00 & 31,00 & 44,00 & 33,60 & 31,00 & 25,00 \\
\hline P10 & 50,00 & 49,40 & 44,00 & 42,70 & 50,00 & 50,00 & 44,00 & 41,40 \\
\hline P20 & 56,00 & 50,00 & 50,00 & 50,00 & 56,00 & 50,00 & 50,00 & 50,00 \\
\hline P30 & 56,00 & 56,00 & 56,00 & 50,00 & 56,00 & 56,00 & 56,00 & 50,00 \\
\hline P40 & 69,00 & 56,00 & 56,00 & 56,00 & 69,00 & 69,00 & 56,00 & 50,00 \\
\hline P50 & 69,00 & 69,00 & 69,00 & 62,50 & 69,00 & 69,00 & 69,00 & 56,00 \\
\hline P60 & 75,00 & 75,00 & 69,00 & 69,00 & 75,00 & 75,00 & 75,00 & 69,00 \\
\hline P70 & 75,00 & 75,00 & 75,00 & 75,00 & 75,00 & 75,00 & 75,00 & 69,00 \\
\hline P80 & 81,00 & 81,00 & 81,00 & 75,00 & 81,00 & 81,00 & 76,20 & 75,00 \\
\hline P90 & 94,00 & 94,00 & 94,00 & 81,00 & 94,00 & 88,80 & 94,00 & 81,00 \\
\hline P95 & 95,50 & 100,00 & 94,00 & 100,00 & 100,00 & 100,00 & 94,30 & 88,80 \\
\hline \multicolumn{9}{|l|}{ Ambiental } \\
\hline P5 & 38,00 & 38,00 & 38,00 & 44,00 & 38,00 & 44,00 & 38,00 & 31,00 \\
\hline P10 & 44,00 & 38,00 & 44,00 & 44,00 & 44,00 & 44,00 & 44,00 & 38,00 \\
\hline P20 & 50,00 & 44,00 & 50,00 & 50,00 & 50,00 & 50,00 & 50,00 & 50,00 \\
\hline P30 & 50,00 & 50,00 & 50,00 & 56,00 & 56,00 & 56,00 & 56,00 & 52,40 \\
\hline P40 & 56,00 & 56,00 & 56,00 & 56,00 & 56,00 & 56,00 & 56,00 & 56,00 \\
\hline P50 & 63,00 & 56,00 & 56,00 & 63,00 & 63,00 & 63,00 & 63,00 & 63,00 \\
\hline P60 & 63,00 & 63,00 & 63,00 & 63,00 & 63,00 & 63,00 & 63,00 & 63,00 \\
\hline P70 & 69,00 & 69,00 & 69,00 & 69,00 & 69,00 & 69,00 & 69,00 & 69,00 \\
\hline P80 & 69,00 & 75,00 & 75,00 & 75,00 & 75,00 & 75,00 & 75,00 & 71,40 \\
\hline P90 & 75,00 & 81,00 & 81,00 & 81,00 & 81,00 & 81,00 & 81,00 & 81,00 \\
\hline P95 & 81,00 & 88,00 & 81,00 & 88,00 & 88,00 & 81,00 & 81,00 & 81,00 \\
\hline
\end{tabular}


Tabla 4. Estadísticos de bondad de ajuste

\begin{tabular}{|ccccccc|}
\hline $\boldsymbol{\chi}^{\mathbf{2} / \mathbf{g l}}$ & CFI & NFI & GFI & AGFI & RMRS & RMSEA \\
$2102,455 / 246=8,55$ & .87 & .85 & .92 & .90 & .043 & $.061(.059-0.64)$ \\
\hline
\end{tabular}

$\mathrm{CFI}=$ Comparative Fix Index; NFI= Normed Fit Index; GFI= Goodness of Fit Index; AGFI= Adjusted Goodness of Fit Index; RMRS = Standardized Root Mean Square Residual; RMSEA= Root Mean Square Error of Approximation.

de la edad, en todos los dominios, con excepción del ambiental, probablemente por el tipo de preguntas que este incorpora (por ejemplo, ¿cuán satisfecho está con su transporte?).

Dentro de las limitaciones del estudio se puede mencionar que la distribución de la muestra considerando la edad presenta diferencias en su composición, así el grupo de personas entre los 20 y 29 años fue más numeroso, lo que podría estar interviniendo en los resultados. Del mismo modo, en este estudio no se evaluó el nivel socioeconómico de los participantes, lo que podría arrojar diferencias en la calidad de vida.

Los hallazgos del estudio hacen visible la necesidad de seguir explorando el comportamiento de este instrumento en términos, por ejemplo, de su estabilidad temporal o como resultado de salud percibido, a fin de ser incorporado como un instrumento adecuado para su uso en la práctica clínica cotidiana, tal como lo sugiere la OMS ${ }^{3}$.

\section{Referencias}

1. WHOQOL Group. The World Health Organization Quality of life assessment (WHOQOL): Position paper from the World Health Organization. Soc Sci Med 1995; 41: 1403-9.

2. Urzúa A, Caqueo-Urízar A. Calidad de Vida, una revisión teórica del concepto. Ter Psicol 2012; 30 (1): 61-71.

3. WHOQOL Group. Development of the WHOQOL, Rationale and current status. Int J Ment Helath 1994; 23 (3): 24-56.

4. WHOQOL Group. The World Health Organization Quality of Life assessment (WHOQoL): development and general psychometric properties. Soc Sci Med 1998; 46 (12): 1569-85.

5. Skevington S, Bradshaw J, Saxena S. Selecting national items for the WHOQOL: concepts and psychometric considerations. Soc Sci Med 1999; 48: 473-87.

6. Li L, Young D, Xiao S, Zhou S \& Zhou L. Psychometric properties of the WHO Quality of life Questionnaire (WHOQOL-100) in patients with chronic diseases and their caregivers in China. Bulletin of the World Health Organization 2004; 82 (7): 493-502.

7. WHOQoL Group. Development of the World Health Organization WHOQoL-Bref Quality of Life Assessment. Psychol Med 1998; 28: 551-8.

8. Skevington S, Lotfy M, O'Conell K. The World Health Organization's WHOQoL-Bref quality of life assessment: Psychometric properties and results of the international field trial. A report from the WHOQOL Group. Qual Life Res 2004; 13: 299-310.

9. Saxena S, Carlson D, Billington R, Orley J. The WHO quality of life assessment instrument (WHOQoL-Bref): The importance of its items for cross-cultural research. Qual Life Res 2001; 10: 711-21.

10. Fang C, Hsiung P, Yu C, Chen M, Wan J. Validation of the World Health Organization quality of life instrument in patients with HIV infection. Qual Life Res 2002; 11: 753-62.

11. Jang Y, Hsieh C, Wang Y, Wu Y. A Validity study of the WHOQoL-Bref Assessment in persons with traumatic spinal cord injury. Arch Phys Med Rehabilitation 2004; 85: $1890-5$.

12. Barros da Silva A, Fleck M, Pechansky F, de Boni R, Sukop P. Psychometric properties OF THE World Health Organization Quality of Life instrument (WHOQoL-Bref) in alcoholic males: A pilot study. Qual Life Res 2005; 14: 473-8.

13. Izutsu T, Tsutsumi A, Islam A, Matsuo Y, Sayuri H, Kurita $\mathrm{H}$, et al. Validity and realiability of the Bangla version of WHOQoL-Bref on a adolescent population in Bangladesh. Qual Life Res 2005; 14: 1783-9.

14. Trompenaars F, Masthoff E, Van Heck G, Hodiamont $\mathrm{P}$, De Vries J. Content validity, construct validity, and reliability of the WHOQoL-Bref in a population of Dutch adult psychiatric outpatients. Qual Life Res 2005; 14: 151-60.

15. Yao G, Wu Ch. Factorial Invariance of the WHOQoLBref among disease groups. Qual Life Res 2005; 14: 1881-8.

16. Berlim M, Pavanello D, Caldieraro M, Fleck M. Reliability and validity of the WHOQoL-Bref in a sample of Brazilian outpatients with major depression. Qual Life 
Res 2005; 14: 561-4.

17. Von Steinbüchel N, Lischetzke T, Gurny M, Eid M. Assessing quality of life in older people: psychometric properties of the WHOQoL-Bref. Eur J Ageing 2006; 3: 116-22.

18. Moreno A, Faerstein E, Werneck G, Lopes C, Chor D. Propiedades psicométricas do instrumento Abreviado de Avaliação de Qualidade de Vida da Organização Mundial da Saúde no Estudo Pró-Saúde. Cad. Saúde Pública 2006; 22 (12): 2585-97.

19. Fleck M, Louzada S, Xavier M, Chachamovich E, Vierira I, Santos L, et al. Aplicação da versão em Português do instrumento abreviado de avaliação da qualidade de vida “WHOQoL-Bref”. Rev Saúde Pública 2000; 34 (2): 178-83.

20. Min S, Kim K, Lee C, Jung Y, Suh S, Kim D. Development of the Korean versions of WHO Quality of Life scale and WHOQoL-Bref. Qual Life Res 2002; 11: 593-600.

21. Noerholm V, Groenvold M, Watt T, Bjorner J, Rasmussen N, Bech P. Quality of life in the Danish general population-normative data and validity of WHOQoLBref using Rasch and item response theory models. Qual Life Res 2004; 13: 531-40.

22. Lucas-Carrasco R. The WHO quality of life (WHOQoL) questionnaire: Spanish development and validation studies. Qual Life Res 2012; 21: 161-5.

23. Urzúa A, Miranda-Castillo C, Caqueo-Urízar A, Mascayano F. Do cultural values affect quality of life evaluation? Soc Indic Res 2013; 114: 1295-1313.

24. Torres M, Quezada M, Rioseco R, Ducci ME. Calidad de vida de adultos mayores pobres de viviendas básicas:
Estudio comparativo mediante uso de WHOQoL-Bref. Rev Med Chile 2008; 136: 325-33.

25. Urzúa A, Castillo P, Gemmel M, Campos C. Relación entre la Orientación a la Comparación Social y el autoreporte de Calidad de Vida. Revista Colegio Psicólogos del Perú 2010; 9 (1): 10-30.

26. Urzúa A, Cortés K, Maita C, Osorio K, Caqueo-Urízar A. La valoración de la importancia en el autoreporte de la Calidad de Vida en la adultez. Rev Med Chile 2013 141: 1010-18.

27. Urzúa A. Calidad de Vida y Factores Biopsicosociales en Patologías Médicas Crónicas. Ter Psicol 2008; 26 (2): 207-14.

28. Urzúa A, Jarne A. Calidad de Vida y Estilos de Afrontamiento en Personas con patologías crónicas. Rev Interam Psicol 2008; 42 (1): 151-60.

29. Espinoza I, Osorio P, Torrejón MJ, Lucas-Carrasco R, Bunout D. Validación del cuestionario de calidad de vida (WHOQoL-Bref) en adultos mayores chilenos. Rev Med Chile 2011; 139: 579-86.

30. World Health Organization. WHOQoL-Bref: Introduction, administration, scoring and generic version of the assessment. Field Trial Version 1996. Programme on mental health. Geneva.

31. Hawthorne $\mathrm{G}$, Herrman $\mathrm{H}$, Murphy B. Interpreting the WHOQoL-Bref: preliminary populations norms and effects sizes. Soc Indic Res 2006; 77: 37-59.

32. Batista JM, Coenders G, Alonso J. Análisis factorial confirmatorio. Su utilidad en la validación de cuestionarios relacionados con la salud. Med Clin (Barc) 2004; 122: 21-7. 